\title{
Complete Chloroplast Genome Sequencing and Genetic Relationship Analysis of Capsicum chinense Jacq
}

\author{
Sebastin Raveendar, Kyung Jun Lee ${ }^{1}$, Myoung-Jae Shin ${ }^{1}$, Gyu-Taek Cho ${ }^{1}$, Jung-Ro Lee ${ }^{1}$, Kyung-Ho Ma, \\ Gi-An Lee ${ }^{1 *}$, Jong-Wook Chung ${ }^{2}$ * \\ ${ }^{1}$ National Agrobiodiversity Center, National Institute of Agricultural Sciences, RDA, Jeonju 54874, Korea \\ ${ }^{2}$ Department of Industrial Plant Science and Technology, Chungbuk National University, Cheongju 28644, Korea
}

\begin{abstract}
Capsicum chinense is one of the five domesticated pepper species belonging to the Solanaceae family. Capsicum sp. have been used as model systems in comparative and evolutionary genomics because their superior availability of chloroplast genome in the solanaceae family. Similarly, molecular markers derived from the complete chloroplast genome can provide effective tools for species identification and phylogenetic resolution. So far however, only partial taxonomic and phylogenetic analyses have been carried out for the genus. Thus, the complete chloroplast genome sequence of a cultivated pepper (C. chinense) has been reported here. The total length of the chloroplast genome is $156,936 \mathrm{bp}$, with $37.7 \%$ overall GC content. A pair of inverted repeats (IRs) of $25,847 \mathrm{bp}$ was separated by a small single copy (SSC) region of $17,912 \mathrm{bp}$ and a large single copy (LSC) region of $87,330 \mathrm{bp}$. The chloroplast genome harbors 113 known genes, including 79 protein-coding genes, four ribosomal RNA genes, and 30 transfer RNA (tRNA) genes. In all, 21 of these genes are duplicated in the inverted repeat regions, 15 genes and six tRNA genes contain a single intron, while two genes have two introns. Analysis revealed 117 simple sequence repeat (SSR) loci, which are mostly located in the intergenic regions. The complete chloroplast genome reported here enriches our knowledge of the genetic complement of $C$. chinense, and contributes to our understanding of the genetic relationships within the genus Capsicum.
\end{abstract}

Keywords Capsicum chinense, Chloroplast, DNA sequencing, Hot pepper

\section{INTRODUCTION}

Capsicum L. (Solanaceae) is one of the most economically important vegetable crops with versatile applications as a food and source of spice, as well as for ornamental and pharmaceutical purposes (Qin et al. 2014). The tropical regions of South America are believed to be the origin of peppers which are now grown worldwide (Greenleaf 1986). Today, the Capsicum genus consists of about 25 wild species and five cultivated species which include $C$. annuum L., C. baccatum L. var. pendulum (Willd.) Eshbaugh., C. chinense Jacq., C. frutescens L., and C. pubescens Ruiz \& Pav. (Kumar et al. 2006). Species identification in
Capsicum is traditionally based on phenotypic characteristics and hybridization studies (Heiser and Smith 1953; Pickersgill 1988; Hunziker 1998; Onus and Pickersgill 2004). However, species identification based on morphological characteristics is often difficult, since most of these features are also influenced by environmental factors. In the last few decades, genotypic molecular markers have been identified, and are gaining increasing importance in resolving phylogenetic relationships.

Capsicum chinense is well known for its distinctive pungency causing severe heat sensation and has been recorded as the hottest pepper in the world (Bosland and Baral 2007). Characterization of genebank accessions is an

Received August 25, 2017; Revised September 8, 2017; Accepted September 8, 2017; Published December 1, 2017

*Corresponding author Gi-An Lee, gknt11@korea.kr, Tel: +82-63-238-4882, Fax: +82-63-238-4859

*Corresponding author Jong-Wook Chung, jwchung73@chungbuk.ac.kr, Tel: +82-43-261-2524, Fax: +82-43-271-0413 
important step for germplasm conservation, maintenance, and breeding studies. Several methodologies have been widely applied to analyze genetic variability in Capsicum species diversity studies. These include using phenotypic markers such as morphological and agronomic descriptors and genotypic markers such as restriction fragment length polymorphism (RFLP) (Lefebvre et al. 1993), amplified fragment length polymorphism (AFLP) (Aktas et al. 2009), random amplified polymorphic DNA (RAPD) (Adetula 2006; Moses and Umaharan 2012), microsatellite or simple sequence repeat (SSR) (Portis et al. 2007; Stagel et al. 2009; Pacheco-Olvera et al. 2012), random amplified microsatellite polymorphism (RAMPO) (Rai et al. 2013) and direct amplification of minisatellite DNA (DAMDPCR) (Ince et al. 2009).

Recently, whole genome sequencing strategies have been used to explore many plant species at the molecular level in rapid and cost-effective ways (Cao et al. 2011). Fragments of DNA from the chloroplast (cp) genome have been widely used for phylogenetic reconstruction and species-level identification because of their relatively stable genomic structure and higher evolutionary rate relative to mitochondrial genomes (Dong et al. 2012). The complete cp genome sequences of cultivated peppers have been reported (Jo et al. 2011; Zeng et al. 2016; Shim et al. 2016; Park et al. 2016; Kim et al. 2016). The main objective of this study was to sequence the complete cp genome and examine the genetic architecture of $C$. chinense with a view to resolving its internal relationships.

\section{MATERIALS AND METHODS}

\section{Sampling and DNA extraction}

The cultivated pepper (Accession No: IT247196) seeds were collected from the National Agrobiodiversity Center, Rural Development Administration, Republic of Korea. Seeds were germinated and grown in a greenhouse, fresh leaves were collected from 40-day-old seedlings and DNA was extracted to construct cp DNA library.

\section{Chloroplast genome sequencing and assembly}

An Illumina paired-end cp DNA library (average insert size of $500 \mathrm{bp}$ ) was constructed using the Illumina TruSeq library preparation kit following the manufacturer's instructions. The library was sequenced with $2 \times 300 \mathrm{bp}$ on the MiSeq instrument at LabGenomics (http://www. labgenomics.co.kr/). Assembly of complete cp genome sequences was performed by de novo assembly of the low coverage whole genome sequence (WGS), via a bioinformatics pipeline (http://www.phyzen.com). Briefly, prior to de novo cp genome assembly, low quality sequences (quality score $<20 ;$ Q20) were filtered out, and the remaining high quality reads were assembled using the CLC Genome Assembler (version beta 4.6, CLC Inc. Aarhus, Denmark) with a 200 - 600-bp overlap size. Cp contigs were selected from the initial assembly by performing a BLAST (ver. 2.2.31) search against known cp sequences. The selected contigs were oriented to construct the complete $\mathrm{cp}$ genome structure. Ambiguous nucleotides or gaps were corrected manually to build the complete cp genome.

\section{Chloroplast genome annotation}

The web-based program Dual OrganellarGenoMe Annotator (DOGMA, http://dogma.ccbb.utexas.edu/) was used to annotate the assembled genome using default parameters to predict protein coding, transfer RNA (tRNA) genes and ribosomal RNA (rRNA) genes. Subsequently, BLASTN (ver. 2.2.31) was used to further identify introncontaining gene positions by searching a published $\mathrm{cp}$ genome sequence (GenBank accession NC_018552). A cp gene map was constructed using the OrganellarGenomeDRAW software (OGDRAW, http://ogdraw.mpimp-golm. mpg.de).

\section{Discovery of SSRs and SNPs}

MIcroSAtellite identification tool (MISA) software (http://pgrc.ipk-gatersleben.de/misa/) was used to find the SSR markers present in the cp genome. This software allows the localization and identification of both perfect and compound microsatellites with 1 to 6 nucleotides in the basic repeat unit. To be considered a microsatellite using this approach, a sequence must be present for a minimum of 10 repeat units for mononucleotide motifs, six repeat units for dinucleotide motifs and five repeat units for all motifs of larger size. MISA has been used for SSR identification in 
many species including Eucalyptus (Ceresini et al. 2005) and barley (Thiel et al. 2003). To identify SNP and InDel variants in the cp genome, we used msaTovcf program locally modified with an in-house script (Page et al. 2016).

\section{Comparative phylogeny}

A new multiple sequence alignment was performed by adding 13 species belonging to the Solanaceae family. The resulting alignment was imported in MEGA 6.0 for inferring maximum parsimony (MP) analysis, setting 1000 bootstrap replications, Tree-Bisection-Regrafting (TBR) algorithm and 10 random addition replicates. All positions containing gaps and missing data were eliminated. Phylogenetic tree was generated by a maximum likelihood (ML) analysis using MEGA 6.0 with 1000 bootstrap replicates (Tamura et al. 2013).

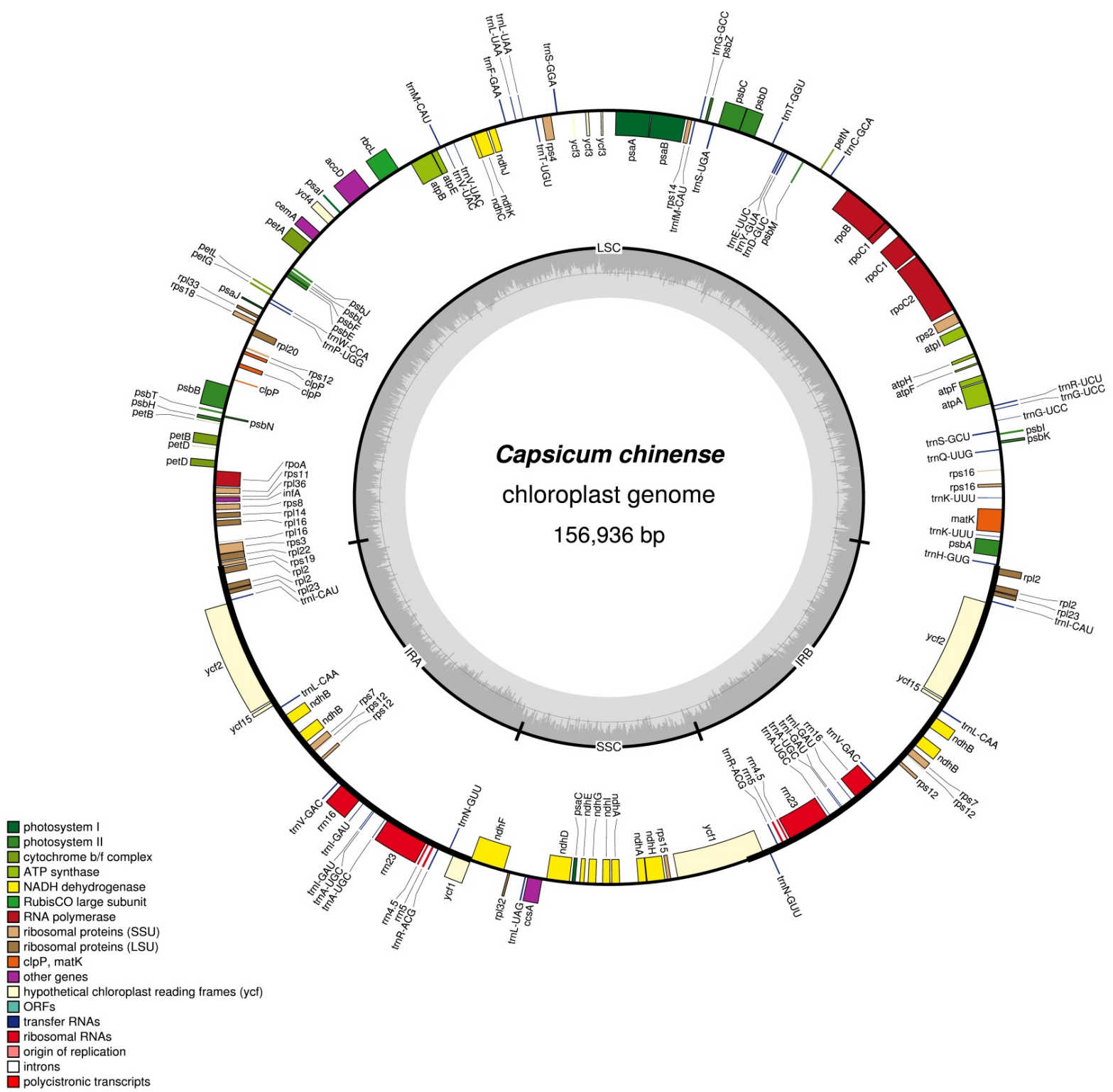

Fig. 1. Complete chloroplast genome map of the cultivated pepper (Capsicum chinense). Genes drawn inside the circle are transcribed clockwise, while those outside are counterclockwise and marked with two arrows. Differential functional gene groups are color-coded. The GC content variation is shown in the middle circle. 


\section{RESULTS}

\section{Chloroplast genome assembly}

Illumina sequencing generated $10,056,140$ paired-end reads, with an average fragment length of $280 \mathrm{bp}$, which were then analyzed to reveal $3,017,719,480 \mathrm{bp}$ of sequence. After removal of low-quality reads (Q20), the remaining high quality reads were mapped to the reference cp genome of $C$. annuum $\mathrm{L}$. (GenBank accession NC_018552). As nucleic DNA was not excluded during DNA extraction, a total of 128,000 cleaned reads were mapped with an average coverage of $173 \times$ on the $\mathrm{cp}$ genome. The cp reads extracted from the Illumina dataset were assembled into a total of four contigs. A complete cp genome was constituted without gaps, from overlaps between contigs (Fig. 1). The whole genome sequencing approach successfully allowed micro-reads to be assembled correctly using a reference-guided method. The fully annotated cp genome sequence of the cultivated pepper $C$. chinense has been deposited in the GenBank database under accession number KX913217.

\section{Features of the chloroplast genome}

The complete cp genome of $C$. chinense is $156,936 \mathrm{bp}$ in length. It has the typical angiosperm plastome structure, including a pair of inverted repeats (IRA and IRB) of $25,847 \mathrm{bp}$ that separate the large single-copy (LSC) region of $87,330 \mathrm{bp}$ from the small single-copy (SSC) region of $17,912 \mathrm{bp}$. The overall GC content of the cp genome is

Table 1. General features of the Capsicum chinense chloroplast genome.

\begin{tabular}{lc}
\hline \hline \multicolumn{1}{c}{ Features } & Chloroplast \\
\hline Genome size (bp) & 156,936 \\
GC content (\%) & 37.7 \\
Total number of genes & 113 \\
Protein coding genes & 79 \\
No. of rRNA genes & 4 \\
No. of tRNA genes & 30 \\
No. of gene duplications in IR regions & 21 \\
Total introns & 17 \\
Single intron (gene) & 15 \\
Double intron (gene) & 2 \\
Single intron (tRNA) & 6 \\
\hline
\end{tabular}

$37.7 \%$, while the LSC and SSC are 35.8 and $32.0 \%$, respectively. The cp genome contains 79 protein coding genes, 30 tRNA genes and four rRNA genes, totaling 113 unique genes (Table 1). Of these, 21 genes are duplicated in the inverted repeat regions, 15 genes and six tRNA genes contain one intron, while two genes ( $y c f 3$ and rps 12) have two introns.

\section{Discovery of SSRs and SNPs}

Cp SSRs (cpSSRs) analyzed with MISA Perl script revealed a total of 117 potential SSRs motifs which are located primarily in the intergenic region (Supplementary Table S1). The majority of the cpSSRs in this cp genome are tri-nucleotides $(58.11 \%)$ and di-nucleotides $(36.75 \%)$. Only six tetra-nucleotides $(5.12 \%)$ were present in the $C$. chinense cp genome. SNP and InDel variants extracted from a multi-FASTA alignment with reference cp genome sequence of C. annuит (JX270811) revealed a total of 174 mutations ( 82 SNPs and 92 InDels) and 69 of these variants involving more than one nucleotide (Supplementary Tables S2 and S3). Among the detected variants, 35 SNPs and $7 \mathrm{InDels}$ were observed in the genic region of the $\mathrm{cp}$ genome.

\section{Phylogenetic analysis}

The phylogenetic relationship between $C$. chinense and other Solanaceae family members was determined by collecting 13 published cp genome sequences from GenBank of the NCBI database (Fig. 2). Resulting alignment and ML phylogenetic tree using MEGA 6.0 strongly indicated that the $C$. chinense was 129-bp larger than the reported $C$. chinense cp genome (KU041709.1) and much closer to C. annuum var. glabriusculum, a wild progenitor of C. annuum, than other Capsicum species. All the nodes in the phylogenetic tree received high bootstrap $(100 \%)$.

\section{DISCUSSION}

Cp sequences have been used to identify different plant species with DNA barcoding methods (Walsh and Hoot 2001; Martine et al. 2006). Furthermore, massively parallel 


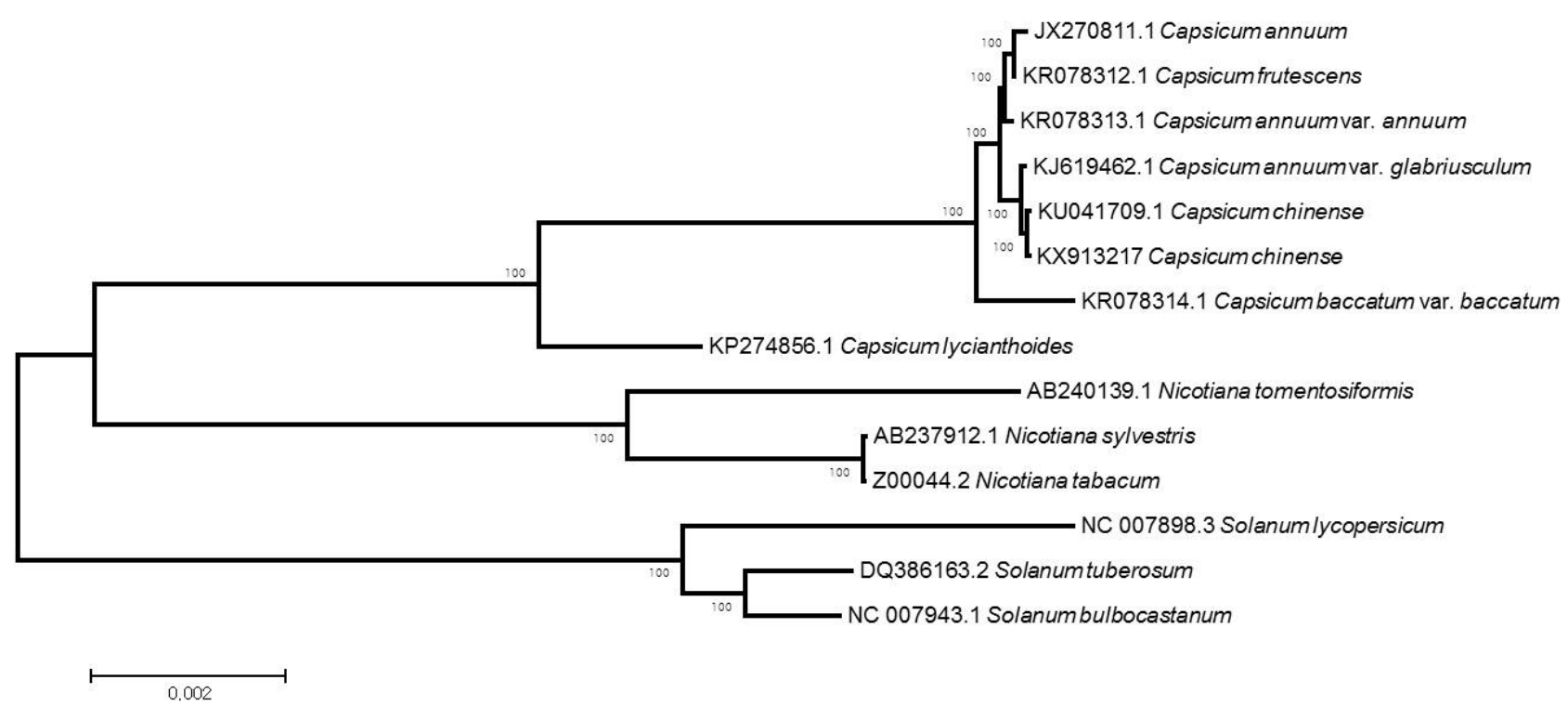

Fig. 2. Maximum likelihood (ML) phylogram of the 13 Solanaceae species using whole chloroplast genome sequences. Numbers above each node indicate the ML bootstrap support values.

Table 2. Genes present in the Capsicum chinense chloroplast genome.

\begin{tabular}{|c|c|}
\hline Group of genes & Name of genes \\
\hline Photosystem I & psaA, psaB, psaC, psaI, psaJ, ycf $3^{\mathrm{y})}, y c f 4$ \\
\hline Photosystem II & $\begin{array}{l}\text { STpsbA, psbB, psbC, psbD, psbE, psbF, psbH, psbI, psbJ, psbK, psbL, psbM, } \\
\quad P p s b N, p s b T, p s b Z\end{array}$ \\
\hline Cytochrome $b 6 / f$ & $\operatorname{pet} A, \operatorname{pet} B^{\mathrm{z})}, \operatorname{pet} D^{\mathrm{z})}, \operatorname{pet} G, \operatorname{pet} L, \operatorname{pet} N$ \\
\hline ATP synthase & $\operatorname{atp} A, \operatorname{atp} B, \operatorname{atp} E, a t p F^{z)}, \operatorname{atpH}, \operatorname{atpI}$ \\
\hline Rubisco & $r b c L$ \\
\hline NADH oxidoreductase & $n d h A^{\mathrm{z})}, n d h B^{\mathrm{z}) \mathrm{x})}, n d h C, n d h D, n d h E, n d h F, n d h G, n d h H, n d h I, n d h J, n d h K$ \\
\hline Large subunit ribosomal proteins & 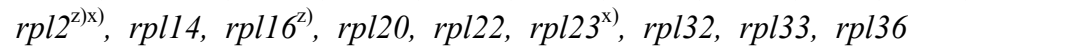 \\
\hline Small subunit ribosomal proteins & $\begin{array}{l}r p s 2, r p s 3, r p s 4, r p s 7^{\mathrm{x})}, r p s 8, r p s 11, r p s 12^{\mathrm{y}) \mathrm{x}) \mathrm{w})}, \operatorname{rps} 14, r p s 15^{\mathrm{x})}, \operatorname{rps} 16^{\mathrm{z})}, r p s 18, \\
\quad r p s 19^{\mathrm{x})}\end{array}$ \\
\hline RNA polymerase & $r p o A, r p o B, r p o C 1^{\mathrm{z})}$, rроC2 \\
\hline Unknown function protein coding gene & $y c f 1^{\mathrm{x})}, y c f 2^{\mathrm{x})}, y c f 15^{\mathrm{x})}$ \\
\hline Other genes & $\operatorname{css} A, \operatorname{cem} A, \operatorname{clp} P 1, \operatorname{clp} P 2, \operatorname{matK}$ \\
\hline Ribosomal RNAs & $r r n 16^{\mathrm{x})}, \operatorname{rrn} 23^{\mathrm{x})}, \operatorname{rrn} 4.5^{\mathrm{x})}, r r n 5^{\mathrm{x})}$ \\
\hline Transfer RNAs & 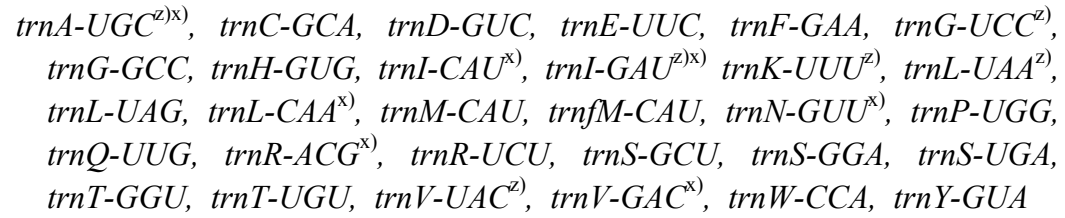 \\
\hline
\end{tabular}

\footnotetext{
${ }^{\mathrm{z})}$ Gene containing a single intron; ${ }^{\mathrm{y})}$ Gene containing two introns; ${ }^{\mathrm{x})}$ Two gene copies in IRs; ${ }^{\text {w) }}$ Trans-splicing gene.
}

sequencing technologies have efficiently increased the phylogenetic resolution of land plants at low taxonomic levels (Parks et al. 2009). In recent years, cp DNA has been considered a promising approach for the study of plant evolution, as many published chloroplast genome sequences are available in the NCBI database. In this study, Illumina re-sequencing and assembly of the $C$. chinense cp genome has an indicated length of 156,936 bp which is 
129-bp larger than the reported $C$. chinense cp genome (156,807 bp). Total GC contents were $37.7 \%$, which is consistent with reported cp genomes of $C$. chinense and other cultivated peppers (Jo et al. 2011; Park et al. 2016;).

$\mathrm{Cp}$ structural rearrangements and gene loss/gain events occur frequently in some angiosperms. The organization and gene architecture of the $C$. chinense cp genome exhibited similar characteristics, with a total of 113 genes as reported in the previous study. However, in the present study only 21 genes were duplicated in IR regions. As shown in Fig. 1 and Table 2, the genome organization appeared to be more conserved among unique genes, as discovered previously in the Capsicum species (Park et al. 2016; Zeng et al. 2016). The conserved gene sequences in the cp genome indicated that maintenance of gene clusters might be essential for normal gene expression. The sequence data generated using the Illumina platform covered a greater depth $(173 \times)$ than has previously been reported, thus, the cp assembly described here with its new insights supports the suggestion of a previous report recommending the significance of greater genome coverage (Wu et al. 2014).

Microsatellite SSRs are widely used as markers in phylogenetic investigations because of their polymorphic nature within the species (Xue et al. 2012). A total of 117 potential cpSSRs were identified in the $C$. chinense cp genome with most of them being intergenic. These results are in agreement with trends of SSR density observed in small genomes (Morgante et al. 2002). Similarly, pairwise comparison of the $C$. chinense cp genome with the reference cp sequence revealed a total of 174 mutations (82 SNPs and 92 InDels) with 69 of these variants involving more than one nucleotide, indicating that they can be used as molecular markers to study the genetic diversity and genetic structure of the Capsicum species.

A new multiple sequence alignment was performed by downloading 13 Solanaceae species from NCBI. Resulting alignment and phylogenetic analysis using MEGA 6.0 indicated $C$. chinense was 129-bp larger than the reported C. chinense cp genome (KU041709.1). As shown in Fig. 2, the constructed ML tree indicated three major clades, Capsiceae, Solaneae, and Nicotianeae, with very high overall bootstrap values and is in agreement with the results of an earlier study (Park et al. 2016).

In conclusion, Illumina sequencing followed by de novo assembly and extensively annotated cp genome of the most important vegetable crop in the Solanaceae family (Capsicum) were reported. The cp genome is wellconserved in terms of size, gene arrangement, and coding sequences, within major subgroups of Capsicum as reported. We also performed comparative analyses with cp genomes sequenced in other Solanaceae species. These analyses revealed that cpSSR sequences were particularly frequent in the $C$. chinense cp genome which is similar to other small plastomes of land plants. Moreover, the ML tree determined the evolutionary position of the pepper in the Solanaceae family. This new genomic dataset will enable further exploration of the genetic diversity of $C$. chinense in the genus Capsicum.

\section{ACKNOWLEDGEMENTS}

This study was carried out with the support of the "Research Program for Agricultural Science \& Technology Development (Project No. PJ010898)" and was supported by the 2016 Postdoctoral Fellowship Program of the National Institute of Agricultural Sciences, Rural Development Administration, Republic of Korea.

\section{REFERENCES}

Adetula OA. 2006. Genetic diversity of Capsicum using random amplified polymorphic DNAs. Afr. J. Biotechnol. 5: $120-122$.

Aktas H, Abak K, Sensoy S. 2009. Genetic diversity in some Turkish pepper (Capsicum annuum L.) genotypes revealed by AFLP analyses. Afr. J. Biotechnol. 8: 4378-4386.

Bosland PW, Baral JB. 2007. 'Bhut Jolokia' - The world's hottest known Chile pepper is a putative naturally occurring interspecific hybrid. Hortscience 42: 222-224.

Cao J, Schneeberger K, Ossowski S, Gunther T, Bender S, Fitz J, et al. 2011. Whole-genome sequencing of multiple Arabidopsis thaliana populations. Nat. Genet. 43: 956-963. Ceresini PC, Silva CLSP, Missio RF, Souza EC, Fischer CN, 
Guillherme IR, et al. 2005. Satellyptus: analysis and database of microsatellites from ESTs of Eucalyptus. Genet. Mol. Biol. 28: 589-600.

Dong W, Liu J, Yu J, Wang L, Zhou S. 2012. Highly variable chloroplast markers for evaluating plant phylogeny at low taxonomic levels and for DNA barcoding. PLoS ONE 7: e35071.

Greenleaf WH. 1986. Pepper breeding. In: MJ. Bassett (ed.), Breeding vegetable crops. AVI Publishing Co., Westport.

Heiser CB, Smith PG. 1953. The cultivated capsicum peppers. Economic Botany 7: 214-227.

Hunziker AT. 1998. Estudios sobre solanaceae. XLVI. Los Ajíes silvestres de argentina (Capsicum). Darwiniana 36: 201-203.

Ince AG, Karaca M, Onus AN. 2009. Development and utilization of diagnostic DAMD-PCR markers for Capsicum accessions. Genet. Res. Crop Evol. 56: 211-221.

Jo YD, Park J, Kim J, Song W, Hur CG, Lee YH, et al. 2011. Complete sequencing and comparative analyses of the pepper (Capsicum annuum L.) plastome revealed high frequency of tandem repeats and large insertion/deletions on pepper plastome. Plant Cell Rep. 30: 217-229.

Kim T-S, Lee J-R, Raveendar S, Lee G-A, Jeon Y-A, Lee H-S, et al. 2016. Complete chloroplast genome sequence of Capsicum baccatum var. baccatum. Mol. Breed. 36: 1-5.

Kumar S, Kumar R, Singh J. 2006. Cayenne/American pepper (Capsicum species). In: KV. Peter (ed.). Handbook of herbs and spices. Woodhead, Cambridge.

Lefebvre V, Palloix A, Rives M. 1993. Nuclear RFLP between pepper cultivars (Capsicum annuum L). Euphytica 71: 189-199.

Martine CT, Vanderpool D, Anderson GJ, Les DH. 2006. Phylogenetic relationships of andromonoecious and dioecious Australian species of Solanum subgenus Leptostemonum section Melongena: Inferences from ITS sequence data. Syst. Bot. 31: 410-420.

Morgante M, Hanafey M, Powell W. 2002. Microsatellites are preferentially associated with nonrepetitive DNA in plant genomes. Nat. Genet. 30: 194-200.

Moses M, Umaharan P. 2012. Genetic structure and phylogenetic relationships of Capsicum chinense. J. Am. Soc. Hortic. Sci. 137: 250-262.

Onus AN, Pickersgill B. 2004. Unilateral incompatibility in Capsicum (Solanaceae): Occurrence and taxonomic distribution. Ann. Bot. 94: 289-295.

Pacheco-Olvera A, Hernandez-Verdugo S, Rocha-Ramirez V, Gonzalez-Rodriguez A, Oyama K. 2012. Genetic diversity and structure of pepper (Capsicum Annuum L.) from Northwestern Mexico analyzed by microsatellite markers. Crop Sci. 52: 231-241.

Page AJ, Taylor B, Delaney AJ, Soares J, Seemann T, Keane JA, et al. 2016. SNP-sites: rapid efficient extraction of SNPs from multi-FASTA alignments. Microbial Genomics 2: $\mathrm{e} 000056$.

Park H-S, Lee J, Lee S-C, Yang T-J, Yoon JB. 2016. The complete chloroplast genome sequence of Capsicum chinense Jacq. (Solanaceae). Mitochondrial DNA Part B 1: 164-165.

Parks M, Cronn R, Liston A. 2009. Increasing phylogenetic resolution at low taxonomic levels using massively parallel sequencing of chloroplast genomes. BMC Biology 7: 84.

Pickersgill B. 1988. The genus Capsicum: a multidisciplinary approach to the taxonomy of cultivated and wild plants. Biologisches Zentralblatt 107: 381-389.

Portis E, Nagy I, Sasvari Z, Stagel A, Barchi L, Lanteri S. 2007. The design of Capsicum spp. SSR assays via analysis of in silico DNA sequence, and their potential utility for genetic mapping. Plant Sci. 172: 640-648.

Qin C, Yu CS, Shen YO, Fang XD, Chen L, Min JM, et al. 2014. Whole-genome sequencing of cultivated and wild peppers provides insights into Capsicum domestication and specialization. Proc. Natl. Acad. Sci. U.S.A. 111: 5135-5140.

Rai VP, Kumar R, Kumar S, Rai A, Kumar S, Singh M, et al. 2013. Genetic diversity in Capsicum germplasm based on microsatellite and random amplified microsatellite polymorphism markers. Physiol. Mol. Biol. Plants 19: 575-586.

Shim D, Raveendar S, Lee JR, Lee GA, Ro NY, Jeon YA, et al. 2016. The complete chloroplast Genome of Capsicum frutescens (Solanaceae). Appl. Plant Sci. 4: 1600002.

Stagel A, Gyurjan I, Sasvari Z, Lanteri S, Ganal M, Nagy I. 2009. Patterns of molecular evolution of microsatellite loci in pepper (Capsicum spp.) revealed by allele sequencing. Plant Syst. Evol. 281: 251-254.

Tamura K, Stecher G, Peterson D, Filipski A, Kumar S. 2013. MEGA6: Molecular Evolutionary Genetics Analysis version 6.0. Mol. Biol. Evol. 30: 2725-2729. 
Thiel T, Michalek W, Varshney RK, Graner A. 2003. Exploiting EST databases for the development and characterization of gene-derived SSR-markers in barley (Hordeum vulgare L.). Theor. Appl. Genet. 106: 411-422.

Walsh BM, Hoot SB. 2001. Phylogenetic relationships of Capsicum (Solanaceae) using DNA sequences from two noncoding regions: The chloroplast $a t p B-r b c L$ spacer region and nuclear waxy introns. Int. J. Plant Sci. 162: 1409-1418.

Wu ZH, Gui ST, Quan ZW, Pan L, Wang SZ, Ke WD, et al. 2014. A precise chloroplast genome of Nelumbo nucifera
(Nelumbonaceae) evaluated with Sanger, Illumina MiSeq, and PacBio RS II sequencing platforms: insight into the plastid evolution of basal eudicots. BMC Plant Biol. 14: 289.

Xue J, Wang S, Zhou SL. 2012. Polymorphic chloroplast microsatellite loci in Nelumbo (Nelumbonaceae). Am. J. Bot. 99: e240-e244.

Zeng FC, Gao CW, Gao LZ. 2016. The complete chloroplast genome sequence of American bird pepper (Capsicum annuиm var. glabriusculum). Mitochondrial DNA A DNA Mapp. Seq. Anal. 27: 724-726. 